\title{
L'ANTHROPOLOGIE, LA COMMUNICATION ET LEURS LIEUX
}

\author{
Jean Lohisse ${ }^{1}$
}

\section{L'anthropologie de la communication}

\section{Une nouvelle perspective}

Dans la théorie classique de la connaissance, l'essai pour expliquer la manière dont nous percevons et connaissons le monde, repose sur un présupposé : le monde existe hors de nous qui l'observons. Dans cette optique, l'univers apparaît comme constitué d'objets. L'objet se définit indépendamment de moi et, plus largement, de tout son environnement. Dans la démarche scientifique, on détermine d'autant mieux sa réalité "objective" qu' on l'isole expérimentalement ("tout autre chose restant égale" dit-on alors). L'objectivité de l'univers des objets tient donc dans leur double indépendance : d'une part, à l'égard de l'observateur humain et d'autre part, vis-à-vis du milieu naturel où ils se trouvent.

1 Professeur au Département de communication de l'Université catholique de Louvain.

Recherches en communication, $\mathrm{n}^{\circ} 12,(1999)$. 
Nous n'avons cependant aucun moyen de prouver que l'objet, indépendant de l'observation, existe. En d'autres termes, nous ne pouvons prouver que la réalité existe indépendamment de nous, observateurs. Il faut simplement l'admettre.

Tout ira bien tant que ce présupposé n'est pas remis en question. Mais ne voilà-t-il pas que d'aucuns ${ }^{1}$, avancent que ce que nous pouvons savoir dépend de l'observateur et non de ce qui est observé. Ce que nous appelons objet est en fait le produit de notre construction individuelle et sociale.

Les constructivistes -vocable sous lequel certains auteurs ont rassemblé ces scientifiques venus d'horizons très divers ${ }^{2}$ - prennent donc le risque d'affirmer que nous construisons ou inventons la réalité plutôt que nous la découvrons. Il est donc erroné, selon eux, de diviser le monde en deux : d'une part, le monde subjectif de notre expérience ; d'autre part, le monde prétendument objectif de la réalité. En fait, l'observateur, le phénomène observé et le processus d'observation sont vus comme formant un tout, tout que l'on ne peut impunément désarticuler.

Cette façon de voir va engendrer bien des idées nouvelles et de nouveaux processus d'analyse tant dans les sciences dites exactes que dans les sciences humaines.

$\mathrm{Si}$ notre regard actif, en ce compris nos paroles et nos comportements d'échange, construit le monde et, en particulier, les autres, nos semblables, on voit immédiatement combien pareille perspective renouvelle bien des théories sur la communication et la connaissance des interactions sociales. Après les thèmes de la mort de l'homme et de l'anti-humanisme théorique qui, avec l'idéologie structuraliste, portaient à son paroxysme la désespérance scientiste, voici le retour du sujet, l'affirmation du contexte, l'insistance sur la mise en commun communicationnelle.

Tous ces éléments vont alimenter les théories de l'énonciation ou des actes de langage en linguistique, les théories interactionnistes en sociologie, l'ethnométhodologie du langage, les courants contemporains en anthropologie de la communication.

${ }^{1}$ Parmi bien d'autres, on trouve Heinz von Foerster, Gregory Bateson, Jean Piaget, Francisco Varela ; pas spécialement des farfelus!

${ }^{2}$ Comme me le fait remarquer Jean Widmer, le terme irait tout aussi bien aux tenants de l'approche classique dont la démarche construit une vision dite scientifique du monde. 
Dans ces derniers courants, le foisonnement aussi bien que la diversité des recherches ne permet pas une inscription classificatoire à l'intérieur d'une science qui répugne, par nature, aux découpages formels et qui, sans renier ses spécificités, se réclame de l'ouverture. Quelques préoccupations récurrentes situent toutefois les bases de l'approche.

Ces préoccupations apparaissent dans les recherches nord-américaines entreprises après 1960 , où se marquent des orientations fort différentes de celles qu'avaient prises les sociologues fonctionnalistes. L'intérêt se détourne des mass-media et de leurs effets pour se centrer sur l'analyse des langages en tant qu'activités sociales. L'effort se portera plus précisément sur l'étude de l'intégration des facteurs situationnels et des processus de communication.

La situation, variable spécifique à chaque communication, est ici essentielle. Tout comme est essentiel l'acte de communication -qui ne se limite pas à une stricte activité linguistique : si l'objectif n'est pas l'étude de la langue au sens saussurien, il n'est pas davantage l'étude des actes de langage (de parole ou de discours) des linguistes pragmaticiens.

Globalement, il s'agit donc de reconstituer l'ensemble des règles, des rites, des normes socioculturels qui font la communication. Ceci est clairement posé dès le début des années 60 et en 1964, Dell Hymes, avec un groupe d'autres chercheurs, peut dessiner les lignes d'un vaste programme :

L'ethnographie ${ }^{1}$ et non la linguistique, la communication et non le langage doivent fournir le cadre de référence au sein duquel la place du langage dans la culture et la société pourra être définie.

1 Lévi-Strauss proposait de distinguer ethnographie (récolte d'observations), ethnologie (classification, interprétation), anthropologie (théorisation). Pour être claires, ces frontières ne se sont pas pour autant imposées. Aujourd'hui, le terme socio-anthropologie semble se répandre en zone francophone; il permet, à tout le moins, de marquer une distance par rapport à l'anthropologie philosophique. 


\section{L'étude des actes de communication}

Georges Mounin ${ }^{1}$ partage avec beaucoup de chercheurs l'idée que les termes de socio-, ethno-, ou anthropolinguisitique sont souvent synonymes et estime que ces approches ne possèdent ni un objet distinct ni une discipline scientifique autonome. L'auteur voit là un bel exemple de travail interdisciplinaire entre la linguistique et la sociologie (ou l'ethnologie et l'anthropologie, ajoute-t-il, dans un rapide amalgame).

Pierre Encrevé, poursuivant la rubrique de Mounin, introduit cependant une distinction bien nécessaire pour éviter de tomber dans le fourre-tout dont on a, par ailleurs, parfois accusé l'anthropologie tout entière. Si l'on peut parler d'une sociolinguistique "en tant que linguistique" qui s'intéresserait aux usagers du langage en société (soit l'acte de l'usager de la langue, soit les relations entre usagers et structures sociales), n'est-il pas tout aussi fondé de parler d'une sociolinguistique "en tant que sociologie" où, comme le suggère Widdowson ${ }^{2}$, la maîtrise des structures linguistiques n'est pas la préoccupation mais bien la mise en œuvre appropriée de ces structures dans des situations sociales réelles. Il serait alors tout indiqué de parler d'anthropologie de la communication puisque, comme dit plus haut, l'attention porte sur l'analyse intrasociale et intraculturelle des problèmes de langage.

La distinction entre actes de langage et actes de communication fut le cadre de la lutte entre deux scientifiques que tout opposait : Noam Chomsky et Dell Hymes. Parti de l'idée d'une ethnographie de la parole où l'on étudierait la communication dans une communauté en observant la façon dont ses membres se servent de leurs ressources verbales et non verbales selon le contexte, Hymes est passé assez vite à une ethnographie de la communication où la description des pratiques langagières des divers groupes socio-culturels devrait conduire à une théorie de la communication en tant que système culturel.

Dans ce passage, l'optique anthropologique s'affirme. La communication s'articule non sur le langage des linguistes mais sur la culture que les anthropologues interprètent comme manière collective de faire, de sentir et de penser. L'attention se focalisera donc sur les

${ }^{1}$ Cf. Universalis, vol.13, 1989, p. 830.

2 D. HYMEs, Teaching language of communication, Oxford, Oxford University Press, 1978. 
normes et les variances culturelles et en particulier sur les rituels, la symbolique sociale, l'imaginaire collectif, les valeurs et les représentations sociales.

Ray Birdwhistell fut un des grands inspirateurs de cette démarche. Dans sa recherche du code de l'interaction sociale, Birdwhistell a travaillé entre autres sur les rituels amoureux et esquissé une analyse du comportement social en termes de codes et de règles : il ne s'agit plus des règles de la langue ou de la parole, mais des règles, des codes donnés à la communication par la société.

L'analyse ne porte plus sur le contenu de l'échange mais sur le système qui a rendu l'échange possible, le système de communication. Birdwhistell conçoit la communication comme un système et insiste sur le fait que, dans le système de communication, les interlocuteurs s'engagent ; l'individu ne communique pas, il prend part à une communication ; il participe. Système très complexe en l'occurrence que l'on ne peut réduire à deux ou trois variables mais qu'il faut traiter en tant que tel. Dans l'optique anthropologique de Birdwhistell, la communication devient alors un processus aussi vaste que la culture :

La culture et la communication sont des termes qui représentent deux points de vue ou deux méthodes de représentation de l'interrelation humaine'.

Aujourd'hui, cette vision s'est bien implantée. Pour Marc Augé, l'objet de l'anthropologie est d'abord et essentiellement l'idée que les autres se font de la relation entre les uns et les autres ${ }^{2}$. Mais le type de relation entretenu par rapport à soi, aux autres et au monde, c'est bien, en définitive, une définition de la culture, objet indiscutable de l'anthropologie depuis ses origines. Si l'on accepte l'adéquation quasi synonymique relation/communication et l'imbrication constitutive de ce couple dans la culture, culture et communication deviennent alors les deux faces réversibles d'un même phénomène, objet de toutes les sollicitudes ethno-anthropologiques.

1 "Kinesics", in International Encyclopedia for the Social Sciences, New York, The Macmillan Co \& The Free Press, Volume 8, 1968, p. 384.

2 M AugÉ, Pour une anthropologie des mondes contemporains, Paris, Aubier, 1994, p. 25. 


\section{Courants de recherche}

Nombreuses sont aujourd'hui les recherches en communication qui se revendiquent ou s'inspirent d'une démarche anthropologique. La personnalité de certains chercheurs, l'équipe dont ils se sont entourés ont fait apparaître des courants à l'intérieur de la préoccupation de l'anthropologie de la communication. Ces courants ont développé un programme plus ou moins délimitable et un cadre conceptuel plus ou moins cohérent. Mais ils restent fluctuants, parfois provisoires, moments dans un ensemble vivant où tout se déplace. Ce que traduit assez bien le terme de courant, plus adéquat en l'occurrence que celui d'école.

Trois courants ont, de façon indéniable, marqué le champ des études en anthropologie de la communication et y ont déposé des jalons essentiels. Les études, tant empiriques que spéculatives de ces programmes portent aujourd'hui leurs fruits dans de nombreuses recherches nouvelles en matière d'interaction en situation. Ce sont l'ethnographie de la communication, conduite par Dell Hymes, l'ethnométhodologie avec Garfinkel lorsqu'elle étudie le langage comme pratique privilégiée à travers laquelle se constitue la vie sociale, et l'interactionnisme symbolique dont Goffman, bien qu'il s'en défende, est le représentant, en matière communicationnelle, le mieux connu aujourd'hui en Europe.

Quels que soient leur importance et leur relatif degré d'élaboration, ces trois problématiques ne peuvent ni ne veulent présenter, à elles seules, l'ensemble des axes théoriques et des concepts fondateurs d'une anthropologie de la communication qui, à l'image même de la communication qu'elle étudie, fonctionne comme un orchestre sans chef (ou, du moins, jusqu'ici, sans un formulateurcoordinateur comme le fut Paul Watzlawick pour Palo Alto) où chaque instrumentiste improvise sur des thèmes incertains.

Vouloir repérer tous ces savants-artistes et leurs thèmes d'interprétation serait une tâche bien difficile et risquée. Au grand danger d'injustice et à titre d'exemples, citons pourtant, dans la sphère européenne et plus spécifiquement française, les recherches sur la sociologie du présent (Morin, de Certeau, Lalive d'Epinay), sur l'ethnologie de la réception (Morley, Lull, Souchon, Lafrance, Mancini) ou encore sur ce que l'on nomme parfois l'ethnologie européenne (Augé, Balandier, Althabe). À remarquer cependant que le centrage communicationnel de ces auteurs est bien souvent loin 
d'être évident. À ceci il convient d'ajouter les problématiques préoccupées de l'approche macroscopique des problèmes anthropologiques de la communication ; ici se situent les démarches de nombreux chercheurs qui se sont attachés à des typologies évolutives où la communication tient une place centrale.

\section{Les lieux de la communication}

\section{Le contexte comme champ social}

L'optique anthropologique marque aujourd'hui la recherche en communication, des philosophes du langage ordinaire aux pragmaticiens, linguistes ou psycho-sociologues. Dans leurs travaux, beaucoup de chercheurs se sont penchés sur ce que, de façon globale, on peut appeler des "micro-univers" : un hôpital, une prison, une école... Ceci tient, au moins en partie, à une tendance générale du moment qui entend privilégier l'ordinaire, le banal, le quotidien, le concret des échanges et des sujets parlants. Mais on peut y voir aussi une claire opposition aux données idéales et abstraites, sans pour autant abandonner la théorie ; on refuse ici d'opposer l'utilité et la raison théorique.

$\mathrm{La}$ façon dont les anthropologues procèdent leur reste cependant spécifique. Une de leurs méthodes favorites, dite parfois "méthode de l'histoire naturelle", est fondée sur l'observation systématique des données recueillies de façon non contrainte, en milieu naturel. C'est une démarche classique en ethnologie, à cette différence près qu'ici, elle porte plutôt sur des contextes que sur des contenus.

Le contexte peut être un environnement sémiotique mais, pour l'anthropologue, c'est avant tout la situation qui importe, ce terme désignant le cadre et les circonstances dans lesquels se déroule une interaction. Il s'agit d'une notion très large où chaque étude de terrain se voit rapidement amenée à opérer des sous-distinctions comme le cadre physique, le scénario, le milieu institutionnel, les rituels... Opération dangereuse toutefois dans la mesure où, fondamentalement, le contexte est un "champ social, ensemble de systèmes symboliques, de structures et de pratiques" 1 .

${ }^{1}$ E. MARC, D. PICARD, L'interaction sociale, Paris, PUF, 1989, p. 84. 


\section{Le local et l'univers}

Ce que Fernand Braudel dit de la durée est tout à fait extrapolable à l'espace -ou mieux, à l'espace-temps. Les faits du temps court (et des micro-espaces) ne sont vraiment intelligibles que si on les inscrit dans la moyenne durée (et un environnement élargi) ; mais la toile de fond reste la longue durée (et l'espace total de la culture et des cultures de l'humanité).

Cette précision ouvre à des questions plus vastes comme celle du rapport du particulier au général, du micro au macro. Selon le poids des faits, on peut voir ainsi des micro-événements qui dans le quotidien banal constituent un ordre social le plus souvent imperceptible aux membres du groupe eux-mêmes et des macroévénements comme les phénomènes institutionnels, ou extraordinaires comme un rite de passage. Les premiers et les autres s'imbriquent intimement dans une culture qui, comme disait Edward Hall, cache plus de choses qu'elle n'en révèle.

Dans l'analyse scientifique, cette interdépendance n'est pas toujours reconnue. Il y a à cela des raisons tendanciellement dogmatiques parce que les positions prises sont porteuses de corollaires politiques. On peut admettre les accentuations ; mais il faut dénoncer l'insuffisance sinon les dangers de sélections ou oppositions qui font bien peu de cas de l'existence et de la complexité de ces rapports. Après le doctrinarisme du positivisme sociologique où le système s'impose aux acteurs, évitons de tomber dans le sectarisme de ceux qui voient dans les actions et stratégies quotidiennes le seul mode d'explication valable. Pour comprendre, la prise en compte de toutes les variables, macro et micro, est requise.

Cette remarque n'épuise évidemment pas le débat. Mais y participer n'est pas notre propos. En ramenant le problème au domaine de l'espace et des lieux de recherche ethnoanthropologiques, l'intention est simplement de proposer quelques distinctions qui ne constituent, en aucun cas, des catégories formelles mais bien plutôt un avertissement à l'encontre de celles-ci.

L'adjectif micro pourrait s'attacher à un lieu déterminé, le local, où, au-delà de la description, la conceptualisation reste proche de l'expérience, là où l'optique macro prendra plus de distance pour ce travail de conceptualisation. Mais il ne peut y avoir de réelle rupture entre les démarches dès lors que le lieu initial s'élargit dans les comparaisons, rapprochements, extrapolations et là, dans la recherche, 
s'insinuent, fut-ce virtuellement, des constantes universelles. Dans son livre Naven, Gregory Bateson ne s'est pas contenté de rapporter, à travers descriptions et entretiens, ses expériences de terrain, au début des années trente, dans quelques tribus de Nouvelle-Guinée. Il a cherché à construire une théorie de la culture qui dépasse de loin le cadre de la société observée. Inutile de multiplier les exemples de ces cheminements de pensée qui, tout en s'ancrant sur le terrain, entendent ne point s'y embourber dans la pure relation factuelle. L'anthropologie n'a-t-elle pas pour tâche délicate de "saisir des concepts qui, pour un autre peuple, relèvent de l'expérience proche, et le faire assez bien pour les placer dans un rapport éclairant avec des concepts éloignés de l'expérience" 1 ?

Dans le prolongement de ce qui précède, deux démarches peuvent être observées dans l'approche anthropologique du lieu. Une question très simple suffit à départager les travaux : le lieu est-il l'objet ou le cadre de la recherche?

A partir de cette interrogation, Christian Bromberger propose de parler d'approche locale et d'approche localisée ${ }^{2}$. Dans le premier cas, le lieu choisi polarise toute l'attention et concentre tout l'effort d'analyse; la prétention ou l'espoir est de parvenir à une compréhension aussi complète que possible à partir de la description minutieuse de ce lieu circonscrit; on peut alors voir là une approche monographique totalisante (au sens où Marcel Mauss parle de phénomène social total). Dans le second cas, celui de l'approche localisée, l'objectif est de faire apparaître des formes générales à partir d'occurrences locales; il importe de s'attacher à la mise au jour des logiques internes qui guident les pratiques, rituels et faits dispersés. Il s'agit ici encore de positionnements différents mais non contradictoires et antagonistes, tant il est vrai que les chercheurs, marchant sur un même terrain, ne peuvent que se croiser et partager leurs expériences. On ne peut séparer collecte et interprétation, inventaire situé et analyse de portée générale. "Faire de l'ethnologie, c'est nourrir sans cesse sa pratique de ce que l'ethnologie fait sur tous les autres terrains ${ }^{3 n}$.

1 C. GEERTZ, Savoir local, savoir global. Les lieux du savoir, Paris, PUF, 1986, p. 74.

2 C. BROMBERGER, "Du grand au petit. Variations des échelles et des objets d'analyse dans l'histoire récente de l'ethnologie de la France", in I. CHIVA, U. JUEGGLE, Ethnologies en miroir, Paris, Maison des sciences de l'homme, 1987, pp. 67-94.

${ }^{3}$ D. FABRE, "L'ethnologue et ses sources", Terrain, nº 7 , octobre 1986, p. 11. 


\section{Les lieux de communication}

"Les lieux sont un peu à l'espace ce que les moments sont au temps" 1 . Ils sont d'abord des points de repères géographiques dans l'espace physique, des données réelles de situation. On les saisit dans la perception de nos sens organiques.

Mais habités par l'homme, les lieux deviennent aussitôt espace symbolique. S'y constitue la représentation des objets mais aussi, et surtout, s'y concrétise la représentation de l'autre dans la rencontre, l'échange, le partage, la négociation, l'occupation, l'usage, l'appropriation, le replis, l'exclusion, la confrontation. L'espace symbolique, c'est aussi celui de la relation sociale où s'organise la société elle-même avec ses conventions, ses structures, ses pouvoirs, ses rites, ses représentations collectives, sa culture. Le lieu géographique, structuré par les dynamiques de relation qui organisent la vie sociale et institutionnelle, est alors, avant tout, lieu symbolique où s'actualise la communication.

Le lieu physique devient ainsi lieu de communication en tant qu'espace structuré par des relations entre sujets, individuels et collectifs. De la perception organique de l'espace naturel, on passe, dans un processus d'interprétation, au champ des significations. Processus éminemment communicationnel puisque la problématique communicationnelle est celle qui donne à l'espace sa dimension symbolique là où se constituent les relations interpersonnelles mais aussi les symboles de l'appartenance sociale et culturelle, objets privilégiés de l'observation anthropologique.

Salle de séjour, habitation, quartier, village ou ville, bureau ou atelier, terroir, territoire, pays, à travers la distribution de l'espace, son aménagement, sa représentation (le centre, la périphérie, l'intérieur, l'extérieur, le proche, le lointain...) se marque la symbolique relationnelle où se mettent en place les repères de l'identité et de l'altérité.

Lieu et communication se font mutuellement. Le village, lieu des distances courtes, génère et est généré par l'oralité. Entre la ville et la scribalité s'est instaurée une indéfectible liaison; ensemble, ils ont construit les hauts lieux du pouvoir. Mégapole et massalité sont

${ }^{1}$ B. LAMIZET, Les lieux de communication, Bruxelles, Mardaga, 1992, p. 31. 
indissociables ; ils diffusent en parfaite complicité les lieux communs de la pensée universelle'.

Mais au lieu vient se combiner le mouvement des parcours. Déplacements, trajets, arrêts, détours, contournements, dévoilent des itinéraires sous-jacents, au niveau de la personnalité, de l'appartenance groupale, de l'identité culturelle. Le lieu serait-il, comme le temps, changeant et destructible?

\section{La crise du lieu}

Une recherche centrée sur les lieux de communication ne peut ignorer la distinction forte qu'Augé désigne par les termes de lieu anthropologique et de non-lieu. Même si l'application au terrain en reste délicate et si sa validité, dans son radicalisme, peut s'y avérer discutable, la proposition ne manque pas d'être stimulante pour l'œil et pour l'esprit. Le lieu peut être considéré comme anthropologique dès lors que l'identité, les relations et l'histoire de ceux qui l'habitent s'y inscrivent dans l'espace. Par opposition, le non-lieu visera les espaces de la circulation, de la distribution et de la communication, où ni l'identité, ni la relation, ni l'histoire ne se laissent appréhender ${ }^{2}$.

Regrettons au passage l'antagonisme terminologique qu'Augé établit entre relation et communication là où, plus haut, j'évoquais la synonymie ; je préfère pour ma part la position de Daniel Bougnoux liant relation et communication et y confrontant l'information ${ }^{3}$-ce dernier terme collant d'ailleurs bien mieux aux idées de circulation et de distribution. Mais la question terminologique reste secondaire face à l'interrogation que soulève Augé. La civilisation universelle contemporaine (dite aussi occidentale, néo-, post-, ou surmoderne) prépare-t-elle l'évanescence du lieu avec, dans son sillage, une crise sans précédent de l'identité individuelle et collective, en perte des repères qui firent son humanité ?

Sans doute n'est-il autorisé de parler de disparition du lieu qu'à un certain niveau d'abstraction théorique. À travers la grille d'interprétation que propose une lecture globale, apparaissent néanmoins

\footnotetext{
${ }^{1}$ Cf. J. LoHISSE, Les systèmes de communication. Approche socio-anthropologique, Paris, A. Colin, 1998.

2 M. AugÉ, Le sens des autres, Paris, Fayard, 1994, pp. 154 et sv.

3 Cf. D. Bougnoux, La communication contre l'information, Paris, Hachette, 1995.
} 
quelques questions premières posées par l'extrême mobilité dans l'espace naturel qui caractérise nos modes de vie aujourd'hui et par l'apparition, contradictoire, d'un tout nouvel espace, global et immobile à nos perceptions, l'espace virtuel ou cyberespace.

Comparée aux sociétés du passé, la vie sociale semble aujourd'hui marquée du sceau de l'infidélité aux choses, de la précarité dans les engagements, de l'éphémérité dans les relations avec les autres et du détachement par rapport aux lieux. Jean Duvignaud y voit "un individu sans appartenance et sans repère"1. À tout le moins peut-on s'interroger sur la modification essentielle du mode d'affirmation de l'identité portant sur le mouvement bien plus que sur l'appartenance et l'identification à des valeurs, à une histoire ou à un lieu. Identité bien particulière par rapport à la définition antérieure, puisqu'en coupure avec le tissu social, espace cellulaire où se replie le microgroupe et bien plus encore, l'individu lui-même.

Cet espace cellulaire trouve son articulation avec la révolution des nouvelles technologies de la communication. Si, avec ces technologies, l'extrême contraction du temps (nano-, pico-, fento- seconde) mène à son étouffement par abolition de la durée de la réflexion et de l'histoire, le rétrécissement de notre planète qu'opère leurs réseaux n'annonce-t-il pas la déterritorialisation des espaces, la délocalisation des activités humaines, la culture planétaire, la dilution des lieux et les voyages immobiles dans des cyberespaces impalpables? L'anthropologue, n'y trouvant plus son lieu, s'évaporera-t-il alors dans cet espace indécis, se faisant, lui aussi, virtuel ?

Peu enclin à la prospective, le chercheur de terrain opte aujourd'hui pour une attitude pragmatique, tantôt se repliant sur le proche, le foyer, la famille, le voisinage où se cultive l'ordinaire et le banal, tantôt jouant le brouillage entre les sphères distinctes du proche et du lointain. Ces positions s'accordent peu aux interrogations de ceux qui voudraient saisir une culture/communication contemporaine qui, virtualisant le lieu et le moment, se dérobe à elle-même.

Pour l'anthropologue qui connaît ses classiques, le lieu de communication devient alors... un nœud dans son mouchoir... comme "chaque fois qu'on laisse quelque chose d'informulé, qu'on consent à laisser cela tel quel, pendant des années, mais en marquant d'un signe d'avertissement la terminologie qu'on utilise ; de telle sorte que ces

${ }^{1} \mathrm{~J}$. DUVIGnAUD, La solidarité, liens de sang et liens de raison, Paris, Fayard, 1986, p. 207. 
termes puissent se dresser non pas comme des palissades, dissimulant l'inconnu aux chercheurs à venir, mais comme des poteaux indicateurs où 1'on puisse lire : inexploré au-delà de ce point"1.

\section{Addendum : la communication de l'anthropologie}

L'anthropologie propose une façon particulière d'examiner, de scruter et, essentiellement, de voir. Mais en se référant à Garfinkel et à l'ethnométhodologie, il serait plus complet d'écrire de voirrapporter. Cet observable-rapportable, Garfinkel le désigne par des termes comme "accountable", "accounting", "account" que 1'on prendra dans le sens de disponibilité, pour un membre de toute organisation courante, d'un ensemble de pratiques localisées c'est-àdire situées dans un cadre déterminé.

Ceci pose un sérieux problème au travail de l'anthropologue tout en en définissant une nouvelle spécificité. En se penchant sur les pratiques sociales et celles de la communication en particulier, le chercheur constate rapidement qu'il ne peut opter valablement pour la position de l'entomologiste et qu'il est observateur-acteur inséré dans la réalité qu'il étudie. Mais il s'aperçoit aussi, à plus ou moins brève échéance, que le savoir peut prendre d'autres formes que celles du discours scientifique.

Le chercheur devient ici porte-parole, le formulateur. Il s'efface tout en restant indispensable. Le texte ne lui appartient pas et le travail est pourtant son œuvre. Il est narrateur et dans cette forme narrative se dévoile la distance prise sur l'écriture du traité. Difficile tâche que d'éviter l'embourbement dans les impasses du scientisme tout en écartant le piège de l'essayisme littéraire. Il s'agit alors, ni plus ni moins, de réconcilier le savoir et la communication, sans confusion de rôle, mais, pour paraphraser Edgar Morin, en refusant que la spécialisation fonctionnelle doive se payer par une parcellisation absurde et que la connaissance continue à se disloquer en mille savoirs ignares.

${ }^{1}$ G. BATESON, Vers une écologie de l'esprit, Liège, Mardaga, 1992, p. 121. 